\title{
Impact of Transduction Scaling Laws on Nanoelectromechanical Systems
}

\author{
Tsoukalas, Konstantinos; Vosoughi Lahijani, Babak; Stobbe, Søren
}

Published in:

Physical Review Letters

Link to article, DOI:

10.1103/PhysRevLett.124.223902

Publication date:

2020

Document Version

Publisher's PDF, also known as Version of record

Link back to DTU Orbit

\section{Citation (APA):}

Tsoukalas, K., Vosoughi Lahijani, B., \& Stobbe, S. (2020). Impact of Transduction Scaling Laws on Nanoelectromechanical Systems. Physical Review Letters, 124(22), [223902].

https://doi.org/10.1103/PhysRevLett.124.223902

\section{General rights}

Copyright and moral rights for the publications made accessible in the public portal are retained by the authors and/or other copyright owners and it is a condition of accessing publications that users recognise and abide by the legal requirements associated with these rights.

- Users may download and print one copy of any publication from the public portal for the purpose of private study or research.

- You may not further distribute the material or use it for any profit-making activity or commercial gain

- You may freely distribute the URL identifying the publication in the public portal 


\title{
Impact of Transduction Scaling Laws on Nanoelectromechanical Systems
}

\author{
Konstantinos Tsoukalas@, ${ }^{*}$ Babak Vosoughi Lahijani®, and Søren Stobbe® \\ Department of Photonics Engineering, DTU Fotonik, Technical University of Denmark, \\ Building 343, DK-2800 Kgs. Lyngby, Denmark
}

(Received 23 December 2019; accepted 6 May 2020; published 5 June 2020)

\begin{abstract}
We study the electromechanical transduction in nanoelectromechanical actuators and show that the differences in scaling laws for electrical and mechanical effects lead to an overall nontrivial miniaturization behavior. In particular, the previously neglected fringing fields considerably increase electrical forces and improve the stability of nanoscale actuators. This shows that electrostatics does not pose any limitations to the miniaturization of electromechanical systems; in fact, in several respects, nanosystems outperform their microscale counterparts. As a specific example, we consider in-plane actuation of ultrathin slabs and show that devices consisting of a few layers of graphene are feasible, implying that electromechanical resonators operating beyond $40 \mathrm{GHz}$ are possible with currently available technology.
\end{abstract}

DOI: 10.1103/PhysRevLett.124.223902

Microelectromechanical systems (MEMS) are prevalent in modern microtechnologies in the form of microphones, gyroscopes, accelerometers, magnetometers, and thermometers, as well as pressure and chemical sensors [1,2]. A central MEMS functionality is the transduction between electromagnetic and mechanical degrees of freedom [3-7]. Electrostatic transducers have found applications ranging from experimental investigations of fundamental physics [8] over microrobotics and nanorobotics [9] to nanophotonics [10,11], and they were proposed as building blocks for adiabatic computing $[12,13]$. Moreover, electric transduction forms the basis of the optoelectromechanics research on, e.g., optical detection of radio waves [14], graphene-based superconductive devices [15], or manipulation of quantum states [16].

A current research frontier is concerned with scaling MEMS to smaller dimensions, i.e., developing nanoelectromechanical systems (NEMS) [17] whose physics is often described via scaling-law arguments based on the parallel-plate capacitor model $[1,2,18-21]$. This downscaling is desirable not only to reduce the footprint and the consumption of materials and energy but also to enable new functionalities. For example, electrochemistry and fluidics set the length scale of electromechanical integration with biological systems [22], and NEMS combined with nanophotonics opens a wealth of opportunities because of the subwavelength nature of the mechanical components [11]. However, NEMS is not merely scaled down MEMS because atomic and quantum effects such as the Casimir effect [23,24], field emission [25], and size dependence of the Young's modulus [26] become significant at the nanoscale. In addition, constraints imposed by applications as well as limitations to nanofabrication technology imply that aspect ratios [27-29] and more generally the design features in NEMS are different from their MEMS

counterparts. For example, in NEMS photonics, these constraints in practice favor an aspect ratio close to unity [11,30-35], which diminishes the transduction calculated in the parallel-plate model [36,37]. This calls for an

(a)

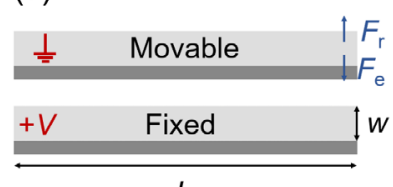

L

(c)

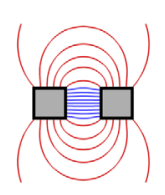

(d)

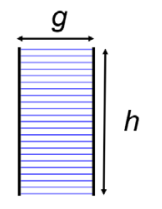

(e)
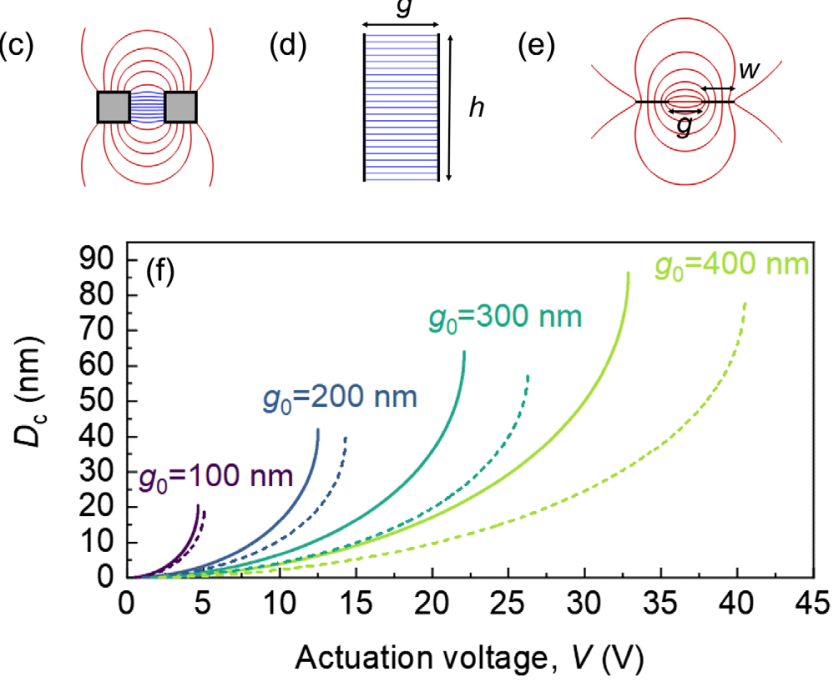

FIG. 1. 3D geometries and 2D electric fields of NEMS electrostatic actuators. Electrostatic actuators consisting of (a) rigid and (b) elastic beams exhibit (c) electric field lines, which combine the characteristics of the fields of a (d) parallel plate and (e) coplanar strips. (f) The displacement of elastic beams calculated with the full electric field (solid) is significantly larger than in the parallel-plate approximation (dashed). 
investigation of electromechanical actuation for arbitrary aspect ratios in MEMS and NEMS, which was missing. Here we consider the impact of scaling laws on the transduction in NEMS actuators for general aspect ratios [Figs. 1(a)-1(e)]. We find that the fringing fields significantly change the scaling laws, instabilities, and actuation range.

We first consider an electrostatic actuator of two rectangular beams [Figs. 1(a) and 1(b)]. The cross-section electric-field lines for an aspect ratio $h=w$, where $h$ is the height and $w$ is the width of the beams, are shown in Fig. 1 (c). The field consists of a combination of the characteristics of the two limiting cases of $h \gg w$, which is a parallel-plate capacitor with a homogeneous field [Fig. 1(d)], and $h \ll w$, which are coplanar strips with a highly inhomogeneous fringing field [Fig. 1(e)].

The displacement, $D_{c}$ at the center of two elastic beams [Fig. 1(b)] is calculated from the Euler-Bernoulli equations with fixed endpoints and subject to an electrostatic pressure $[6,38]$. The latter is calculated from the capacitance per unit length, $C_{2 \mathrm{D}}$, which we derive from the capacitance of a rectangle near a ground plane as [39-41],

$$
C_{2 \mathrm{D}}=\frac{\epsilon_{0}}{2}\left[\frac{2 h}{g}+c\left(\frac{2 w}{g}\right)^{a}+d\left(\frac{h}{w}\right)^{b}+f\right]
$$

where $\epsilon_{0}$ is the vacuum permittivity, $g$ is the cross-sectional distance, $a=b=0.23$ are scaling coefficients, $c=3.31$, $d=0.73, f=-1.06$. Equation (1) is formally valid for $h / g \geq 0.05$ and $0.05 \leq w / g \leq 5$, but we find that it agrees with detailed numerical models beyond these ranges [41]. The first term in Eq. (1) corresponds to a parallel-plate capacitor, the second describes the fringing fields, and the rest do not contribute to the force in this geometry. To illustrate the impact of the fringing fields, we consider silicon beams with width $w=230 \mathrm{~nm}$, height $h=220 \mathrm{~nm}$, length $L=15 \mu \mathrm{m}$, and Young's modulus $E=169 \mathrm{GPa}$, which are experimentally realistic parameters for photonic NEMS $[30,35,42]$. We calculate $D_{c}$ for different initial gaps, $g_{0}$, with and without the fringing fields [Fig. 1(f)]. Notably, displacements are significantly higher than predicted by the inaccurate yet commonly employed parallelplate approximation, e.g., it is approximately two times higher for $g_{0}=100 \mathrm{~nm}$ and a voltage of $V=4.65 \mathrm{~V}$.

The maximum displacement for electrostatic actuators is only a fraction of the initial gap due the pull-in instability [5,43-45]. If the applied voltage is increased beyond a critical value, $V_{p}$, the mechanical restoring force cannot counterbalance the electrostatic force and the two bodies collapse onto each other. Generally, any force that scales as an inverse power law,

$$
F \propto \frac{1}{\left(g_{0}-y\right)^{\nu}}
$$

and is balanced by a linear restoring force has a maximum actuation range of $g_{0} /(\nu+1)$ [31,41]. For example, a parallel-plate actuator has $\nu=2$ and is limited to displacements of $1 / 3$ the initial gap. An important finding of our work, which is already apparent from Fig. 1(f), is that the fringing fields increase the actuation range beyond the parallel plate instability point $g_{0} / 3$. This advantage of low aspect ratio NEMS originates from the scaling laws of the electrostatic force.

In order to study the general behavior of electrostatic actuation, we use a lumped spring-mass model composed of two rigid parallel beams, where one beam is fixed and the other one is attached to a spring [Fig. 1(a)]. The electromechanical force on the movable beam is [41]

$$
F=\frac{\epsilon_{0} L}{2}\left[\frac{h}{\left(g_{0}-y\right)^{2}}+\frac{2^{a-1} w^{a} c a}{\left(g_{0}-y\right)^{a+1}}\right] V^{2}-k y,
$$

from which we calculate the pull-in displacement for three different width to initial gap ratios [Fig. 2(a)]. We observe that the pull-in distances lie between those of the limiting cases of $h \gg w\left(y_{p}=g_{0} / 3\right)$ and $h \ll w\left(y_{p}=g_{0} / 2.23\right)$ in accordance with Eq. (2). In addition, the pull-in voltage is
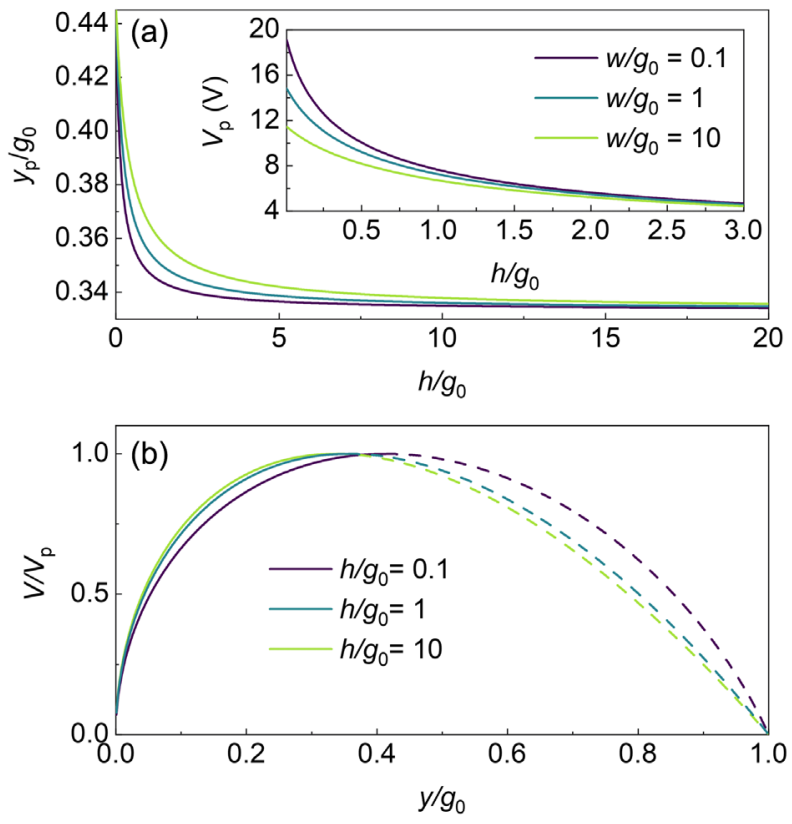

FIG. 2. Displacement and instability in parallel-beams transduction. (a) Normalised pull-in displacement of a rigid parallelbeam actuator [Fig. 1(a)] for different ratios of height to initial gap. The parallel-plate approximation is valid for electrostatic actuators with high aspect ratios and the normalized pull-in distance $y_{p} / g_{0}$ approaches $1 / 3$ for large beam heights. Inset: Pull-in voltage for different height to gap and width to gap ratios. The spring constant is $k=0.5 \mathrm{Nm}^{-1}$ for all widths and heights. The beam width has a significant contribution to the pull-in voltage. (b) Stable (solid) and unstable (dashed) equilibrium curves for different ratios of height to initial gap, $h / g_{0}$, and $w=g_{0}$. 
much larger for flat actuators $\left(h \ll g_{0}\right)$ as shown in the inset of Fig. 2(a). We note that the pull-in voltage depends on the spring constant $k$, which in actual devices may depend on $h$ and $w$ but is kept constant in Fig. 2(a) to enable a direct comparison. In contrast, the pull-in displacement and normalized voltage [Fig. 2(b)] are independent of the spring constant [41]. Notwithstanding the importance of the increased pull-in displacement for flat actuators, the most striking conclusion from Fig. 2 is that the quantity of most immediate experimental importance, namely the voltagedisplacement relation [Fig. 2(b)], is only weakly dependent on the aspect ratio. This indicates that very thin electrostatic actuators are feasible thanks to the fringing fields taking over the actuation from the homogeneous fields.

Motivated by the apparent feasibility of actuators with ultrasmall aspect ratios, we investigate this further using the lumped spring-mass model for the limiting case of two coplanar strips. This model can describe ultrathin membranes [46] and 2D materials [47] for which in-plane electrical transduction has never been investigated. The capacitance per unit length of two coplanar strips is given by $C_{s}=\epsilon_{0} K\left(\sqrt{1-q^{2}}\right) / K(q)$ [48-50], where $q=$ $g /(2 w+g)$ and $K$ is the complete elliptic integral of the first kind. The normalized pull-in displacement as a function of width to initial gap ratio is calculated numerically [41] in Fig. 3. The actuation range is always greater than $40 \%$ of the initial gap, and we prove that it asymptotically reaches $1 / 2$ [41], showing that actuation of $2 \mathrm{D}$ beams outperforms those of finite height. The actuation range behaves nonmonotonically for very small width to gap ratios. This is explained by the nontrivial dependence of the capacitance on the width, which in this case, but also in other 2D geometries, is asymptotically logarithmic [41]. Finally, the voltage-displacement dependence (inset in Fig. 3) shows a qualitative behavior of the coplanar strip actuator that is similar to that of the parallel-plate actuator.

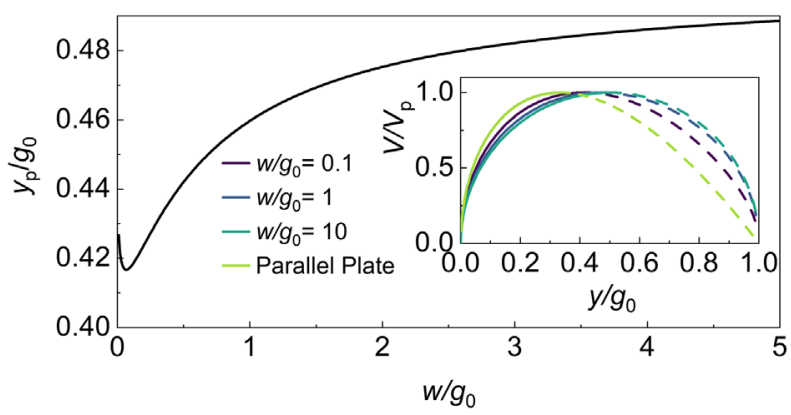

FIG. 3. Normalised pull-in displacement of a coplanar strip actuator for different ratios of width to initial gap. The system corresponds to a parallel-beam actuator of zero height with a cross section shown in Fig. 1(f). Inset: stable (solid) and unstable (dashed) equilibrium curves for different strip width to initial gap ratios. The actuation range is greater than that of a parallel-plate actuator due to the scaling of the fringing forces.
As a concrete example of in-plane actuation mechanisms, we consider two parallel coplanar strips of a suspended graphene monolayer with $w=450 \mathrm{~nm}, L=7 \mu \mathrm{m}$, and $g_{0}=100 \mathrm{~nm}$. The mechanical properties of graphene [51] are characterized by the Young's modulus $E=1 \mathrm{TPa}$, the density $\rho=2200 \mathrm{~kg} \mathrm{~m}^{-3}$, and the sheet height $h=0.35 \mathrm{~nm}$. For a doubly clamped beam, the lumped parameter $k$ is given by $k=32 E h(w / L)^{3}$, which leads to a spring constant of $k=2.98 \mathrm{Nm}^{-1}$. The maximum actuation is found from Fig. 3 to be $x_{p}=49 \mathrm{~nm}$ and the pull-in voltage is calculated to be $V_{p}=20 \mathrm{~V}$ [41]. The fundamental frequency of a beam with a negligible built-in tension is $f_{0}=1.03 w \sqrt{E / \rho} / L^{2}$, which predicts an actuation frequency of $200 \mathrm{MHz}$. These numbers suggest that in-plane actuation can be used for monolithic graphene NEMS that can take full advantage of the material properties and represent the ultimate limit of 2D devices [52], although previous research has focused on out-of-plane actuation. For given beam dimensions, the in-plane to out-of-plane resonance frequency scales as $f_{\text {in }} / f_{\text {out }}=w / h$, indicating orders of magnitude difference in oscillations of $2 \mathrm{D}$ materials. The highest frequency demonstrated via out-of-plane actuation is $f_{\text {out }}=1.17 \mathrm{GHz}$ [53] for a beam of $w=3 \mu \mathrm{m}$ and $L=1.2 \mu \mathrm{m}$, which was achieved by taking advantage of high built-in tension. However, the out-of-plane frequency of this resonator with zero built-in-tension is calculated to be $f_{\text {out }}=5.3 \mathrm{MHz}$. If the same beam is actuated in plane, the frequency is $f_{\text {in }}=45.7 \mathrm{GHz}$, which shows that in-planeactuated nanomechanical devices may operate at speeds consistent with modern optical communication systems. Most importantly, our analysis shows that in-plane actuation of $2 \mathrm{D}$ materials is indeed possible and enables reconfigurable 2D quantum-dot networks [54] and ultrathin tunable metamaterials $[55,56]$.

We finally turn to comb-drive actuators [Fig. 4(a)], which are employed because of their linear capacitancedisplacement relationship, enabling large displacements without a pull-in instability in the actuation direction [57,58]. Traditionally, comb-drive actuators have been treated in the parallel-plate approximation, even in devices with near-unity aspect ratios [13,28,33,34,59]. The electric fields of a periodic cell in the finger array [Fig. 4(b)] can be classified in three categories with distinct impact on the actuation force: fields created by the finger tips, $E_{f}$, fields between overlapping fingers, $E_{p}$ and $E_{s}$, and all remaining fields, $E_{g}$. The fields $E_{p}$ and $E_{s}$ correspond to the parallel plate and coplanar strip fields of the parallel beams. Their capacitance increases linearly with finger overlap, $C=C_{c} s$, where $C_{c}$ is the $2 \mathrm{D}$ capacitance of the cross section and $s$ is the overlap, i.e., it gives rise to a constant force. The field $E_{f}$ encounters only a translational shift in the displacement direction without experiencing any redistribution in its profile and thus $\partial C_{f} / \partial s=0$, meaning that it does not contribute to the force. The field lines of $E_{g}$ give 

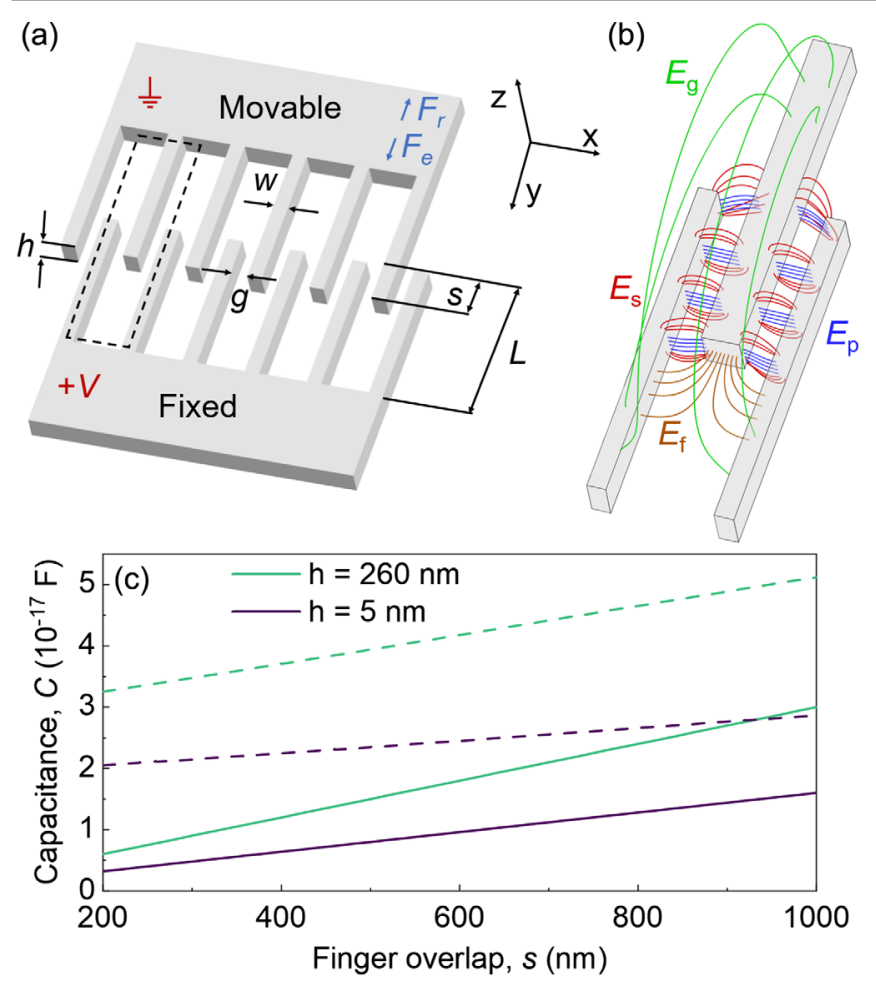

FIG. 4. Electric fields and capacitances of comb-drive actuators. (a) Illustration of a comb-drive actuator. (b) Selected field lines for a unit cell. (c) Capacitance of a unit cell as a function of finger overlap for a near unity aspect ratio $(h=260 \mathrm{~nm})$ as well as for nearly flat slabs $(h=5 \mathrm{~nm})$. The full $3 \mathrm{D}$ capacitance is higher than its 2D approximation as it includes the extra capacitance of the tip fields $E_{f}$ and the global fields $E_{g}$, but it has a lower slope because this extra capacitance has a negative contribution to the force. This capacitance does not change the linear behavior of the actuator that persists in the ultrathin membrane regime.

rise to global forces with no counterpart in parallel plates, beams, and coplanar strips. They connect nonoverlapping regions and terminate on top and/or bottom surfaces, i.e., they can only be understood in a 3D model. These fields are diminished by actuation due to the reduction of the nonoverlapping region, leading to a negative contribution to the force, $\partial C_{g} / \partial s \leq 0$, which tends to disengage the fingers. Previous work [60] approximated the global force as $F_{g}=-4 \epsilon_{0} V^{2}(w+g) /(4 \pi s)$, which does not depend on the height and confirms their out-of-plane character. However, it also predicts a nonlinear dependence on the overlap, but, as we show below, this nonlinearity vanishes for low aspect ratios.

While the separation of field components is instructive for understanding the underlying physics of comb-drive actuators, it is not entirely rigorous, and we therefore use a finite element method to calculate the capacitance of a unit cell as a function of finger overlap for fingers with width $w=250 \mathrm{~nm}$, gap $g=350 \mathrm{~nm}$, length $L=2 \mu \mathrm{m}$, and two different heights of $h=260 \mathrm{~nm}$ and $h=5 \mathrm{~nm}$.
We calculate the 2D cross-section capacitance $C_{c}$ and the full 3D capacitance $C$. The latter is compared to the approximation $C=C_{c} s$ [Fig. 4(c)]. We find that the full 3D capacitance is a linear function of the finger overlap, contrary to the global-force formula in Ref. [60]. The contributions from the fingertip and the global fields make the $3 \mathrm{D}$ capacitance always higher, but its slope always lower, than the 2D approximation. To quantify the global forces, we extract the 3D differential capacitance from a linear fit and compare it to the 2D simulation and the parallel-plate approximation (see Table I). We assume that the difference in the 2D and the 3D calculations is caused by contribution of the global forces, which is quantified to be $\Delta C_{g} / \Delta y=-0.6 \times 10^{-11} \mathrm{Fm}^{-1}$ for both $h=260 \mathrm{~nm}$ and $h=5 \mathrm{~nm}$. This suggests that the global forces take place between the outer surfaces of the fingers and do not depend on their height, in accordance with Ref. [60]. These contributions reduce the total electrostatic force by $20 \%$ for $h=260 \mathrm{~nm}$ and $38 \%$ for $h=5 \mathrm{~nm}$. Finally, we note that the parallel-plate approximation significantly underestimates the electrostatic force of actuators with an aspect ratio near unity and completely fails for ultrasmall aspect ratios, concealing the fact that comb-drive actuators can be used monolithically in ultrathin devices.

From the preceding discussions, it is clear that the forces from the finger width increase in importance with the reduction of height, impacting the miniaturization of the lateral dimensions of the comb-drive actuator. The width of a comb with $N$ finger pairs is $2 N(w+g)$, and we are interested in choosing these parameters such that the actuation force is maximum for a given comb width. Neglecting 3D effects, the figure of merit that must be maximized is $C_{c} /(g+w)$. In the limit of ultrathin actuators, we approximate the cross-sectional capacitance as that of coplanar strips $C_{c}=C_{s}$. In this case, the force depends strongly on the width to gap ratio and is maximum for $w \approx 0.4 g$ [41] and $g$ as small as possible. For finite height, the fingers are approximated as parallel beams with $C_{c}=$ $C_{2 \mathrm{D}}$ given by Eq. (1), which is maximized when both $g$ and $w$ are as small as possible. This is, however, not feasible because of an instability that collapses individual fingers with too small width, which was studied in the parallelplate approximation in Ref. [58]. We linearize the contribution of the capacitance Eq. (1) in the Euler-Bernoulli

TABLE I. Differential capacitance calculated by linear fitting of the capacitance simulations shown in Fig. 4(c).

\begin{tabular}{lcc}
\hline \hline & \multicolumn{2}{c}{$\Delta C / \Delta y\left(10^{-11} \mathrm{~F} \mathrm{~m}^{-1}\right)$} \\
\cline { 2 - 3 } Approximation & $h=260 \mathrm{~nm}$ & $h=5 \mathrm{~nm}$ \\
\hline Parallel plate & 1.3 & 0.025 \\
Simulation 2D $\left(C_{c}\right)$ & 3.0 & 1.6 \\
Simulation 3D & 2.4 & 1.0 \\
\hline \hline
\end{tabular}


equation and introduce the fringing field corrections to the model of Ref. [58] to obtain [41]

$$
\tilde{V}^{2}=V^{2} \frac{\epsilon_{0} L^{4}}{E w^{3}}\left(\frac{24}{g^{3}}-\frac{6.578 w^{0.23}}{h g^{2.23}}\right)
$$

Equation (4), along with the requirement that $\tilde{V} \leq 3.516$, restricts the comb-drive parameters so that there is no instability. For example, for silicon with $V=30 \mathrm{~V}$, $g=350 \mathrm{~nm}, h=260 \mathrm{~nm}$, and $L=2 \mu \mathrm{m}$, the minimum finger width is $w \geq 35 \mathrm{~nm}$. If the fringing field contribution is ignored and a parallel-plate model is used instead, the minimum width is $w \geq 32 \mathrm{~nm}$, showing that the fringing field only has a small effect on the instability as expected from its scaling.

Our findings show that, contrary to prevalent assumptions, NEMS transducers with ultralow aspect ratios are not only electromechanically possible, they are in fact in many ways superior to their counterparts with high aspect ratios. In such flat actuators, the fringing fields take over from the parallel fields, resulting in qualitatively similar transduction but with improved pull-in instabilities. This surprisingly advantageous scaling to flat structures shows that a new realm of nanoelectromechanical experiments and devices is within experimental reach. For example, flat devices enable electromechanical actuation at tens of gigahertz of $2 \mathrm{D}$ materials [52,61], Floquet topological photonics [62], tunable optomechanical crystal resonators [37] or quantum optomechanics based on superconducting resonators [63]. Mechanical oscillators with frequencies in the gigahertz range are of special technological importance, enabling microwave to optical photon converters [64], quantum memories [65], and laser cooling mechanisms [66,67]. Finally, the fact that actuators with ultralow aspect ratios maintain a large capacitance has important implications for the scaling of nanoscale sensors, not only because the capacitance is larger than previously believed but more importantly because in-plane transduction offers ample opportunities for reducing and engineering the stray capacitance, which is the limiting factor in state-of-theart NEMS capacitive sensors [36].

We gratefully acknowledge financial support from the Villum Foundation Young Investigator Program Grant No. 13170.

*Corresponding Author. ktso@dtu.dk

[1] M. J. Madou, Fundamentals of Microfabrication and Nanotechnology (CRC Press, Taylor \& Francis Group, Boca Raton, FL, 2011), Vol. III.

[2] S. D. Senturia, Microsystem Design (Springer, Boston, MA, 2004).
[3] W. C. Tang, T.-C. H. Nguyen, M. W. Judy, and R. T. Howe, Electrostatic-comb drive of lateral polysilicon resonators, Sens. Actuator A 21, 328 (1990).

[4] C. C. Nguyen, MEMS technology for timing and frequency control, IEEE Trans. Ultrason. Ferroelectr. Freq. Control 54, 251 (2007).

[5] J. A. Pelesko and D. H. Bernstein, Modeling MEMS and NEMS (CRC Press, Boca Raton, FL, 2002).

[6] M. I. Younis, E. M. Abdel-Rahman, and A. Nayfeh, A reduced-order model for electrically actuated microbeambased MEMS, J. Microelectromech. Syst. 12, 672 (2003).

[7] P. M. Osterberg and S. D. Senturia, M-TEST: A test chip for MEMS material property measurement using electrostatically actuated test structures, J. Microelectromech. Syst. 6, 107 (1997).

[8] L. Tang, M. Wang, C. Y. Ng, M. Nikolic, C. T. Chan, A. W. Rodriguez, and H. B. Chan, Measurement of non-monotonic Casimir forces between silicon nanostructures, Nat. Photonics 11, 97 (2017).

[9] M. Sitti, Microscale and nanoscale robotics systems [Grand Challenges of Robotics], in Proceedings of the 2004 American Control Conference (IEEE, 2007), Vol. 14, p. 53, https://doi.org/10.1109/MRA.2007.339606.

[10] H. Du, F. Chau, and G. Zhou, Mechanically-tunable photonic devices with on-chip integrated MEMS/NEMS actuators, Micromachines 7, 69 (2016).

[11] L. Midolo, A. Schliesser, and A. Fiore, Nano-opto-electromechanical systems, Nat. Nanotechnol. 13, 11 (2018).

[12] A. Galisultanov, Y. Perrin, H. Samaali, H. Fanet, P. Basset, and G. Pillonnet, Contactless four-terminal MEMS variable capacitor for capacitive adiabatic logic, Smart Mater. Struct. 27, 084001 (2018).

[13] S. O. Erbil, U. Hatipoglu, C. Yanik, M. Ghavami, A. B. Ari, M. Yuksel, and M. S. Hanay, Full Electrostatic Control of Nanomechanical Buckling, Phys. Rev. Lett. 124, 046101 (2020).

[14] T. Bagci, A. Simonsen, S. Schmid, L. G. Villanueva, E. Zeuthen, J. Appel, J. M. Taylor, A. Sørensen, K. Usami, A. Schliesser, and E.S. Polzik, Optical detection of radio waves through a nanomechanical transducer, Nature (London) 507, 81 (2014).

[15] P. Weber, J. Güttinger, I. Tsioutsios, D. E. Chang, and A. Bachtold, Coupling graphene mechanical resonators to superconducting microwave cavities, Nano Lett. 14, 2854 (2014).

[16] J. J. Viennot, X. Ma, and K. W. Lehnert, Phonon-NumberSensitive Electromechanics, Phys. Rev. Lett. 121, 183601 (2018).

[17] M. Roukes, Nanoelectromechanical systems face the future, Phys. World 14, 25 (2001).

[18] S. Baglio, S. Castorina, and N. Savalli, Scaling Issues and Design of MEMS (John Wiley \& Sons, NY, 2008).

[19] D. J. Griffiths, Introduction to Electrodynamics, 4th ed. (Cambridge University Press, Cambridge, England, 2017).

[20] H. C. Nathanson, W. E. Newell, R. A. Wickstrom, and J. R. Davis, The resonant gate transistor, IEEE Trans. Electron Devices 14, 117 (1967).

[21] V. Leus and D. Elata, Fringing field effect in electrostatic actuators, Technical Report TECHNION Israel Institute of Technology, Faculty of Mechanical Engineering, 2004. 
[22] A. Grayson, R. Shawgo, A. Johnson, N. Flynn, Y. Li, M. Cima, and R. Langer, A BioMEMS review: MEMS technology for physiologically integrated devices, in Proc. IEEE (IEEE, 2004), Vol. 92, p. 6, https://doi.org/10.1109/JPROC .2003 .820534 .

[23] H. B. Chan, V. A. Aksyuk, R. N. Kleiman, D. J. Bishop, and F. Capasso, Quantum mechanical actuation of microelectromechanical systems by the casimir force, Science 291, 1941 (2001).

[24] K. Y. Fong, H.-K. Li, R. Zhao, S. Yang, Y. Wang, and X. Zhang, Phonon heat transfer across a vacuum through quantum fluctuations, Nature (London) 576, 243 (2019).

[25] A. M. Loveless and A. L. Garner, Scaling laws for gas breakdown for nanoscale to microscale gaps at atmospheric pressure, Appl. Phys. Lett. 108, 234103 (2016).

[26] H. Sadeghian, C. K. Yang, J. F. L. Goosen, A. Bossche, U. Staufer, P. J. French, and F. V. Keulen, Effects of size and defects on the elasticity of silicon nanocantilevers, J. Micromech. Microeng. 20, 064012 (2010).

[27] E. Sarajlić, M. J. de Boer, H. V. Jansen, N. Arnal, M. Puech, G. Krijnen, and M. Elwenspoek, Advanced plasma processing combined with trench isolation technology for fabrication and fast prototyping of high aspect ratio MEMS in standard silicon wafers, J. Micromech. Microeng. 14, S70 (2004).

[28] K. Takahashi, E. Bulgan, Y. Kanamori, and K. Hane, Submicrometer comb-drive actuators fabricated on thin single crystalline silicon layer, IEEE Transactions on Industrial Electronics 56, 991 (2009).

[29] K. Akarvardar, D. Elata, R. Parsa, G. C. Wan, K. Yoo, J. Provine, P. Peumans, R. T. Howe, and H. Wong, Design considerations for complementary nanoelectromechanical logic gates, in 2007 IEEE International Electron Devices Meeting (IEEE, 2007), p. 299, https://doi.org/10.1109/ IEDM.2007.4418930.

[30] C. Papon, X. Zhou, H. Thyrrestrup, Z. Liu, S. Stobbe, R. Schott, A. D. Wieck, A. Ludwig, P. Lodahl, and L. Midolo, Nanomechanical single-photon routing, Optica 6, 524 (2019).

[31] W. Winger, T. D. Blasius, T. P. Mayer Alegre, A. H. SafaviNaeini, S. Meenehan, J. Cohen, S. Stobbe, and O. Painter, A chip-scale integrated cavity-electro-optomechanics platform, Opt. Express 19, 24905 (2011).

[32] Y. Akihama and K. Hane, Single and multiple optical switches that use freestanding silicon nanowire waveguide couplers, Light Sci. Appl. 1, e16 (2012).

[33] C. Errando-Herranz, N. L. Thomas, and K. B. Gylfason, Low-power optical beam steering by microelectromechanical waveguide gratings, Opt. Lett. 44, 855 (2019).

[34] S. Han, T. J. Seok, C. K. Kim, R. S. Muller, and M. C. Wu, Multicast silicon photonic MEMS switches with gapadjustable directional couplers, Opt. Express 27, 17561 (2019).

[35] K. V. Acoleyen, J. Roels, P. Mechet, T. Claes, D. V. Thourhout, and R. Baets, Ultracompact phase modulator based on a cascade of NEMS-operated slot waveguides fabricated in silicon-on-insulator, IEEE Photonics J. 4, 779 (2012).
[36] M. A. Cullinan, R. M. Panas, C. M. Dibiasio, and M. L. Culpepper, Scaling electromechanical sensors down to the nanoscale, Sens. Actuator A 187, 162 (2012).

[37] H. Pfeifer, T. Paraïso, L. Zang, and O. Painter, Design of tunable $\mathrm{GHz}$-frequency optomechanical crystal resonators, Opt. Express 24, 11407 (2016).

[38] E. Chan, K. Garikipati, and R. Dutton, Characterization of contact electromechanics through capacitance-voltage measurements and simulations, J. Microelectromech. Syst. 8, 208 (1999).

[39] W. C. Chuang, C. W. Wang, W. C. Chu, P. Z. Chang, and W. C. Hu, The fringe capacitance formula of microstructures, J. Micromech. Microeng. 22, 025015 (2012).

[40] Y. Y. Iossel, E. S. Kochanov, and M. G. Strunskii, in The Calculation of Electrical Capacitance, edited by W.W. Kennedy (Foreign Technology Div. Wright-Patterson Afb, Ohio, 1971).

[41] See Supplemental Material at http://link.aps.org/ supplemental/10.1103/PhysRevLett.124.223902 for detailed derivations and further discussions.

[42] K. E. Grutter, M. I. Davanço, K. C. Balram, and K. Srinivasan, Invited Article: Tuning and stabilization of optomechanical crystal cavities through NEMS integration, APL Photonics 3, 100801 (2018).

[43] W. M. Zhang, H. Yan, Z. K. Peng, and G. Meng, Electrostatic pull-in instability in MEMS/NEMS: A review, Sens. Actuator A 214, 187 (2014).

[44] J. I. Seeger and S. B. Crary, Stabilization of electrostatically actuated mechanical devices, in Proceedings of International Solid State Sensors and Actuators Conference, Vol. 2 (IEEE, Chicago, IL, USA, 1997), p. 1133, https://doi.org/10.1109/SENSOR.1997.635402.

[45] J. A. Pelesko and A.A. Triolo, Nonlocal problems in MEMS device control, J. Eng. Math. 41, 345 (2001).

[46] A. Castellanos-Gomez, V. Singh, H. S. J. v. d. Zant, and G. A. Steele, Mechanics of freely-suspended ultrathin layered materials, Ann. Phys. (Berlin) 527, 27 (2015).

[47] C. N. Lau, W. Bao, and J. Velasco, Properties of suspended graphene membranes, Mater. Today 15, 238 (2012).

[48] G. L. Pedrola, in Beam Propagation Method for Design of Optical Waveguide Devices (John Wiley and Sons, NY, 2015), p. 351.

[49] D. F. Lawden, Elliptic Functions and Applications (Springer-Verlag, New York, 1989).

[50] J. D. Jackson, in Classical Electrodynamics, 3rd ed. (Wiley, N.J., 1999), p. 88.

[51] J. S. Bunch, A. M. v. d. Zande, S. S. Verbridge, I. W. Frank, D. M. Tanenbaum, J. M. Parpia, H. G. Craighead, and P. L. Mceuen, Electromechanical resonators from graphene sheets, Science 315, 490 (2007).

[52] D. Garcia-Sanchez, A. M. van der Zande, A. S. Paulo, B. Lassagne, P. L. McEuen, and A. Bachtold, Imaging mechanical vibrations in suspended graphene sheets, Nano Lett. 8, 1399 (2008).

[53] M. Jung, P. Rickhaus, S. Zihlmann, A. Eichler, P. Makk, and C. Schönenberger, $\mathrm{GHz}$ nanomechanical resonator in an ultraclean suspended graphene $\mathrm{p}-\mathrm{n}$ junction, Nanoscale 11, 4355 (2019). 
[54] C. Chakraborty, N. Vamivakas, and D. Englund, Advances in quantum light emission from 2D materials, Nanophotonics 8, 2017 (2019).

[55] N. I. Zheludev and E. Plum, Reconfigurable nanomechanical photonic metamaterials, Nat. Nanotechnol. 11, 16 (2016).

[56] Z. Ren, Y. Chang, Y. Ma, K. Shih, B. Dong, and C. Lee, Leveraging of MEMS technologies for optical metamaterials applications, Adv. Opt. Mater. 8, 1900653 (2020).

[57] J. D. Grade, H. Jerman, and T. W. Kenny, Design of large deflection electrostatic actuators, J. Micromech. Microeng. 12, 335 (2003).

[58] D. Elata and V. Leus, How slender can comb-drive fingers be? J. Micromech. Microeng. 15, 1055 (2005).

[59] X. Chew, G. Zhou, F. S. Chau, J. Deng, X. Tang, and Y. C. Loke, Dynamic tuning of an optical resonator through MEMS-driven coupled photonic crystal nanocavities, Opt. Lett. 35, 2517 (2010).

[60] W. A. Johnson and L. K. Warne, Electrophysics of micromechanical comb actuators, J. Microelectromech. Syst. 4, 49 (1995).

[61] J. Güttinger, A. Noury, P. Weber, A. M. Eriksson, C. Lagoin, J. Moser, C. Eichler, A. Wallraff, A. Isacsson, and A.
Bachtold, Energy-dependent path of dissipation in nanomechanical resonators, Nat. Nanotechnol. 12, 631 (2017).

[62] K. Fang, Z. Yu, and S. Fan, Realizing effective magnetic field for photons by controlling the phase of dynamic modulation, Nat. Photonics 6, 782 (2012).

[63] J. D. Teufel, T. Donner, D. Li, J. W. Harlow, M. S. Allman, K. Cicak, A. J. Sirois, J. D. Whittaker, K. W. Lehnert, and R. W. Simmonds, Sideband cooling of micromechanical motion to the quantum ground state, Nature (London) $\mathbf{4 7 5}$, 359 (2011).

[64] J. Bochmann, A. Vainsencher, D. D. Awschalom, and A. N. Cleland, Nanomechanical coupling between microwave and optical photons, Nat. Phys. 9, 712 (2013).

[65] T. A. Palomaki, J. W. Harlow, J. D. Teufel, R. W. Simmonds, and K. W. Lehnert, Coherent state transfer between itinerant microwave fields and a mechanical oscillator, Nature (London) 495, 210 (2013).

[66] F. Marquardt and S. M. Girvin, Optomechanics, Physics 2, 40 (2009).

[67] J. M. Taylor, A. S. Sørensen, C. M. Marcus, and E.S. Polzik, Laser Cooling and Optical Detection of Excitations in a LC Electrical Circuit, Phys. Rev. Lett. 107, 273601 (2011). 